\title{
Zodiacal exoplanets in time - XIII. Planet orbits and atmospheres in the V1298 Tau system, a keystone in studies of early planetary evolution
}

\author{
E. Gaidos ${ }^{\oplus, 1,2,3 \star}$ T. Hirano ${ }^{\oplus, 4,5,6}$ C. Beichman, ${ }^{7}$ J. Livingston ${ }^{\circledR},{ }^{8}$ H. Harakawa, ${ }^{9}$ K. W. Hodapp, ${ }^{10}$
} M. Ishizuka, ${ }^{8}$ S. Jacobson, ${ }^{10}$ M. Konishi, ${ }^{11}$ T. Kotani,${ }^{4,5,6}$ T. Kudo, ${ }^{9}$ T. Kurokawa, ${ }^{4,12}$ M. Kuzuhara,,${ }^{4,5}$ J. Nishikawa, ${ }^{5,6,4}$ M. Omiya, ${ }^{4,5}$ T. Serizawa, ${ }^{5,12}$ M. Tamura, ${ }^{4,5,8}$ A. Ueda ${ }^{4,5}$ and S. Vievard ${ }^{9}$

${ }^{1}$ Department of Earth Sciences, University of Hawai'i at Mānoa, Honolulu, HI 96822, USA

${ }^{2}$ Center for Space and Habitability, University of Bern, Gesellschaftsstrasse 6, CH-3012 Bern, Switzerland

${ }^{3}$ Institute for Astrophysics, University of Vienna, Türkenschanzstrasse 17, A-1180 Vienna, Austria

${ }^{4}$ Astrobiology Center, 2-21-1 Osawa, Mitaka, Tokyo 181-8588, Japan

${ }^{5}$ National Astronomical Observatory of Japan, NINS, 2-21-1 Osawa, Mitaka, Tokyo 181-8588, Japan

${ }^{6}$ Department of Astronomical Science, School of Physical Sciences, The Graduate University for Advanced Studies (SOKENDAI), 2-21-1, Osawa, Mitaka, Tokyo, 181-8588, Japan

${ }^{7}$ IPAC, Caltech, Pasadena, CA 91125, USA

${ }^{8}$ Department of Astronomy, Graduate School of Science, The University of Tokyo, 7-3-1 Hongo, Bunkyo-ku, Tokyo 113-0033, Japan

${ }^{9}$ Subaru Telescope, 650 N. Aohoku Place, Hilo, HI 96720, USA

${ }^{10}$ University of Hawaii, Institute for Astronomy, 640 N. Aohoku Place, Hilo, HI 96720, USA

${ }^{11}$ Faculty of Science and Technology, Oita University, 700 Dannoharu, Oita 870-1192, Japan

${ }^{12}$ Institute of Engineering, Tokyo University of Agriculture and Technology, 2-24-16, Nakacho, Koganei, Tokyo, 184-8588, Japan

Accepted 2021 October 20. Received 2021 October 19; in original form 2021 August 30

\begin{abstract}
Studies of planetary systems of stars in star-forming regions and young clusters open a window on the formative stages of planetary evolution. We obtained high-cadence high-resolution infrared spectroscopy of the solar-mass Taurus associationmember V1298 Tau during a transit of its $10 R_{\oplus}$-size ' $b$ ' planet. We measured the systemic radial velocity (RV) and find that the kinematics of V1298 Tau suggest an affiliation with a $\gtrsim 6$ Myr-old subgroup. A comparison of V1298 Tau and the nearby, co-moving star 2M0405 with stellar evolution models suggests an age of $\sim 10-25$ Myr. We measured the projected spin-orbit angle of ' $\mathrm{b}$ ' as $\lambda=15_{-16}^{+15}$ and $\lambda=2_{-4}^{+12}$ degrees using the apparent RV shift and change in line profile, respectively, induced by the transient occultation of the rotating star by the planet. These values indicate a prograde orbit like that of the interior ' $c$ ' planet of V1298 Tau and point to a co-planar multiplanet system that formed within a disc. We also measured variation in the strength of the $1083 \mathrm{~nm}$ triplet of neutral orthohelium as a probe of any extended/escaping atmosphere around 'b'. We detect a steady decrease in absorption over the transit that appears to arise from the star or its planetary system. While this variation could be ascribed to ' $b$ ' or possibly to the immediately preceding transit of 'd', we cannot rule out that this is due to rapid variation in the stellar disc-integrated flux in the triplet. The amplitude of variation $(\sim 0.04 \mathrm{~nm})$ is consistent with moderate estimates of atmospheric escape driven by XUV radiation from the central star.
\end{abstract}

Key words: techniques: spectroscopic-Sun: UV radiation - planets and satellites: atmospheres - planets and satellites: physical evolution - stars: activity-planetary systems.

\section{INTRODUCTION}

Observations of planets around young stars provide 'snapshots' of planetary evolution with which we can test models of changes in planet orbits, atmospheres, and compositions. Around the youngest stars (ages of $\leqslant 10 \mathrm{Myr}$ ), many of which retain primordial circumstellar discs, the process of planet formation itself can be probed. Pushing the planetary clock back to ages approaching $1 \mathrm{Myr}$ is seen as crucial given evidence that planet formation proceeded rapidly in the Solar System (e.g. Lee \& Halliday 1996; Kruijer et al. 2017),

^E-mail: gaidos@hawaii.edu and theoretical models proposing that the accretion of some types of planets can occur rapidly (e.g. Johansen \& Lambrechts 2017; Ormel 2017).

The youngest nearby region of ongoing star formation is the Taurus Molecular Cloud, which contains representatives of all the earliest stages of star formation, including Class 0 (deeply embedded), $0 / 1$, and 1 (emergent protostar) sources with inferred model ages $<1 \mathrm{Myr}$ (Froebrich 2005). Taurus is also highly structured (Kraus \& Hillenbrand 2008; Joncour et al. 2018; Galli et al. 2019), with multiple groups with distinct age spanning $\sim 5 \mathrm{Myr}$, and a more disperse population of older stars of poorly determined ages, but which could be at least 10 Myr old (Krolikowski, Kraus \& Rizzuto 2021). Confirmation of earlier detections of giant planets around 
Taurus stars with the radial velocity (RV) method (Donati et al. 2016; Johns-Krull et al. 2016; Yu et al. 2017) has proven elusive (Damasso et al. 2020; Donati et al. 2020). Direct detection by imaging has yielded companions with brown dwarf masses (Itoh et al. 2005; Luhman et al. 2009), while a candidate super Jupiter-mass planet embedded within a disc (Kraus et al. 2012) remains controversial (Thalmann et al. 2015; Mendigutía et al. 2018; Currie et al. 2019). ${ }^{1}$

Space photometry missions, i.e. K2 and TESS, have opened an additional avenue for the detection of young planetary systems by the transit method. Follow-up ground- or space-based spectroscopy during a transit can detect a planet's atmosphere in transmission, and the partial occultation of the disc of the rotating star produces a change in the shape and location of its rotationally broadened spectral lines, constraining the angle between the stellar spin axis and orbit plane. These phenomena may be especially pronounced for young systems where a planet's atmosphere is escaping under the influence of the active star, and the star is rapidly rotating and its lines broad and well resolved. The proliferation of echelle spectrographs operating in the infrared allows such observations to be made at higher signal-to-noise ratios for low-mass pre-main-sequence stars like those in Taurus with $T_{\text {eff }}$ near $4000 \mathrm{~K}$. They also provide access to the $1083 \mathrm{~nm}$ triplet line of neutral orthohelium, which has been used to detect extended and escaping atmospheres (e.g. Nortmann et al. 2018; Spake et al. 2018).

$K 2$ observed part of Taurus during Campaign 4 and David et al. (2019a) reported a Jupiter-size $\left(10.2 \pm 0.6 \mathrm{R}_{\oplus}\right)$ planet on a 24.12-d orbit transiting the solar-mass Taurus member V1298 Tau. Subsequent analysis of those data revealed three additional transiting planets, two interior to and one exterior to the orbit of V1298 Tau b (David et al. 2019b). The radii of these planets is anomalously large compared to counterpart multiplanet systems around middle-aged ( $\gtrsim 1$ Gyr) tars, suggesting that the light-element dominated envelopes of these planets are heated or still hot, and/or the atmospheres are escaping. Feinstein et al. (2021) report observations of the 'c' planet of V1298 Tau and constrain the spin-orbit angle $\lambda$ to $5 \pm 15 \mathrm{deg}$ (a prograde orbit). We obtained high-resolution echelle IR spectra of a transit of V1298 Tau b to (a) measure the 'Doppler' shadow of the planet in the shape of the spectral lines and to search for anomalous absorption in the He I triplet.

\section{OBSERVATIONS AND DATA REDUCTION}

An updated linear ephemeris for V1298 Tau b (T. David personal communication) predicts a transit that was partially visible from Maunakea Observatory with a mid-transit time $T_{c}$ at $\mathrm{BJD}=$ 2459119.0664 (UT 13:36 on 26 September 2020); however, there are significant transit time variations (TTVs) due to mutual perturbations between the planets of V1298 Tau (Livingston et al. in preparation). A fit to a TTV model predicts $T c$ at BJD $=2459119.0231$ or 62 minutes earlier at UT 12:33 with an uncertainty of $1.7 \mathrm{~min}$. We monitored V1298 Tau with the InfraRed Doppler (IRD) spectrograph (Tamura et al. 2012; Kotani et al. 2018) on the 8.2-m Subaru telescope on Maunakea. We obtained 41 spectra of V1298 Tau beginning at first contact (T1), with 3 before T1, and 41 within the $6.42 \mathrm{~h}$ span between T1 and last contact (T4). Monitoring concluded at the end of the night and 31 min before T4. We also obtained 97 other spectra outside of transit to generate a template spectrum which is free from the telluric-line features for our RV analysis. IRD covers $970-1730 \mathrm{~nm}$

${ }^{1}$ Gaidos et al. (submitted) recently reported a Taurus star with one or two super-Jupiter-mass companions. with $\lambda / \Delta \lambda \approx 70000$. Integration times were 8 min during and near the transit. Using the IRAF echelle package (Tody 1986) and a custom reduction pipeline (Hirano et al. 2020a), we extracted onedimensional spectra after flat-fielding and scattered light subtraction. Wavelengths were calibrated using the comparison spectra of the ThAr lamp as well as the laser-frequency comb (LFC) taken during each run. Typical signal-to-noise ratios (S/Ns) at $1000 \mathrm{~nm}$ were $75-115$.

\section{ANALYSIS AND RESULTS}

\subsection{Systemic RV}

The RV analysis for NIR spectra taken by IRD is described in detail in Hirano et al. (2020a). In short, we first generated a high-SNR template spectrum of V1298 Tau that are free of telluric features, by removing telluric features and combining multiple observed IRD spectra taken in different epochs. Individual spectra were then fitted using this template spectrum, taking into account the instantaneous instrumental profile (IP) of the spectrograph inferred from the simultaneously observed LFC's spectra. RVs were extracted for each small spectral 'segment' with a wavelength range that was chosen to cover at least a few stellar absorption lines. For V1298 Tau, having a relatively large rotation velocity $\left(v \sin i>20 \mathrm{~km} \mathrm{~s}^{-1}\right)$, a typical segment length of $2-3 \mathrm{~nm}$ was adopted. The typical RV internal error for each 8-min integration was $34-45 \mathrm{~m} \mathrm{~s}^{-1}$. The mean systemic barycentric $\mathrm{RV}$ is $+14.644 \pm 0.136 \mathrm{~km} \mathrm{~s}^{-1}$.

\subsection{Taurus membership}

Using parallaxes and proper motions from the Gaia EDR3 data release (Gaia Collaboration et al. 2021) and the RV determined from IRD, we calculate $X Y Z=(-98.4 \pm 0.2,+12.26 \pm 0.03$, $-42.86 \pm 0.01) \mathrm{pc}$ and $U V W=(12.68 \pm 0.01,-6.35 \pm 0.02$, $-9.07 \pm 0.01$ ) $\mathrm{km} \mathrm{s}^{-1}$, using left-handed coordinates (positive $X$ and $U$ towards the Galactic Centre). The star is clearly associated with the Taurus cluster, but falls immediately outside the central core of the cluster. Comparing these to the position and kinematics of the sub-groups identified by Krolikowski et al. (2021), the dispersed V1298 Tau is kinematically indistinguishable from the 'D3-South' group among the Taurus sub-groups identified by Krolikowski et al. (2021), but lies distinctly in the foreground of the catalogued members (Fig. 1). Krolikowski et al. (2021) catalogue the star as a member of 'D2'; this sub-group is a younger, more condensed group. There is significant spatial overlap of D3-South with the Group 29 identified by Luhman (2018), and the centroid kinematics are very similar (Fig. 1). Estimates of their ages, however, differ markedly: $6.2_{1.7}^{1.4}$ Myr for D3-South, but with a significantly older ( 13 Myr) component Krolikowski2021, and between the ages of the $\beta$ Pictoris and Tucana-Horologium moving groups (20-45 Myr) for Group 29 (Luhman 2018).

\subsection{Multiplicity, mass, and age}

We revisited the properties of the host star by fitting stellar atmosphere models to available photometry, i.e. from the Paunzen (2015) catalog of Stromgren-Crawford $u b v y \beta$ photometry, APASS BVgri, Tycho $B_{T} V_{T}$, Gaia $G B_{p} R_{p}$, 2MASS $J H K_{s}$, and WISE W1-4. The best-fitting model to 26 data points $\left(\chi^{2}=12.5\right.$ for 18 degrees of freedom) has $T_{\text {eff }}=4900 \mathrm{~K}, \log \mathrm{g}=3.0, \mathrm{Fe} / \mathrm{H}=0$. A Bayesian analysis marginalizing over all models yields average values of $T_{\text {eff }}$ $=4911 \mathrm{~K}$ and $\log g=3.57$. The inferred integrated flux combined with the Gaia parallax yields a luminosity of $0.903 \pm 0.002 \mathrm{~L}_{\odot}$ and 


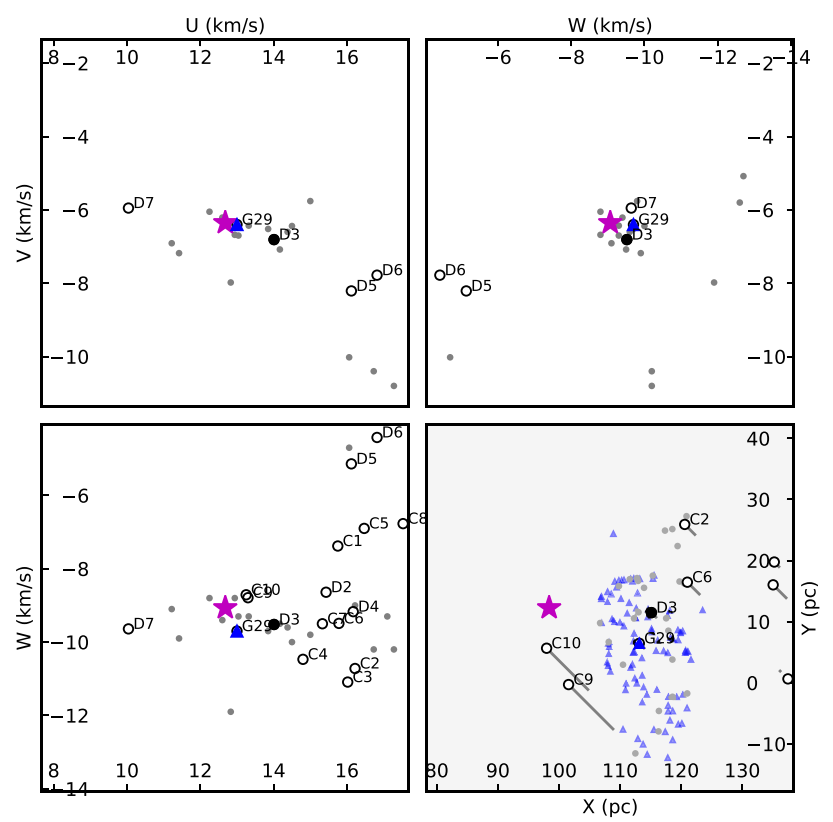

Figure 1. Upper left-hand and right-hand and lower left-hand panels: $U V W$ space motion of V1298 Tau relative to some of the Taurus groups catalogued by Krolikowski et al. (2021) and Group 29 (blue triangle) identified by Luhman (2018). We identify the dispersed D3-South sub-group (filled circle) as the most likely host group. Lower right: Position of V1298 Tau relative to those groups in Galactic Cartesian coordinates. Galactic $Z$ relative to V1298 Tauis represented as the length of the 'pins'. Individual members of the D3-South group and Group 29 are plotted as grey and blue triangles ( $U V W$ are not available for the latter). Coordinate systems are left-handed, i.e. positive $U$ and $X$ towards the Galactic Center.

a radius of $1.317 \pm 0.027 \mathrm{R}_{\odot}$, consistent with but more precise than David et al. (2019a). David et al. (2019a) found a rotation period of $2.865 \pm 0.012 \mathrm{~d}$ that gives an equatorial speed of $23.2 \pm 0.5 \mathrm{~km} \mathrm{~s}^{-1}$, consistent within errors with the $v \sin i$ measured from optical echelle spectra (David et al. 2019a; Feinstein et al. 2021) and indicating that the stellar rotation axis lies close to the plane of the sky.

We compared the $L_{*}$ and $T_{\text {eff }}$ of V1298 Tau to three different sets of models: the BHAC-15 model (Baraffe et al. 2015) which do not account for the effects of stellar activity; the Dartmouth models with (Feiden 2016) and without (Dotter et al. 2008) the effect of magnetic fields that inflate low-mass on the pre-main sequence; and the SPOTS models (Somers, Cao \& Pinsonneault 2020), which account for the effects of spots and their magnetic fields with different coverage fraction (Fig. 2). This comparison points to a mass of 1.07-1.22 $\mathrm{M}_{\odot}$, depending on model, and an age of $8-23 \mathrm{Myr}\left(\delta \chi^{2}\right.$ or 95 per cent confidence for 2 degrees of freedom), which is intermediate between those assigned to the D3-South group and Group 29, with the heavily spotted and magnetic models yield older ages, as expected. While the D3-South age is based on a comparison with BHAC-15 models (Krolikowski et al. 2021), the Group 29 models are based on a comparison with isochrone fits to other co-moving groups, and differences between group member selection, models and fitting procedures might underlie some of the discrepancy.

Simultaneous fitting of multiple co-eval stars, the basis of isochrone age-dating of stellar clusters, can significantly improve mass and age estimates compared to fits of individual stars. In the case of Taurus this is hindered by the region's young age and significant substructure and age heterogeneity. Companion stars formed from the same molecular cloud core are more likely to be

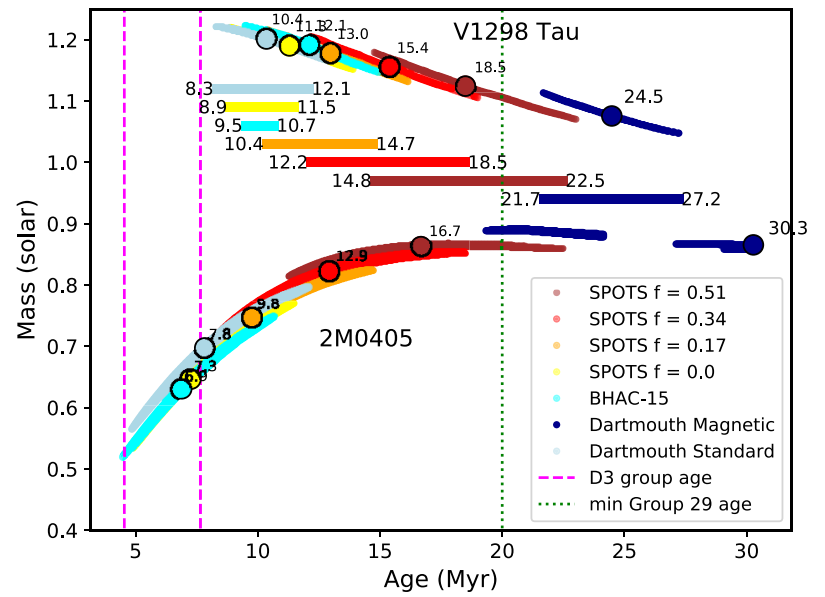

Figure 2. Masses and ages of Dartmouth (Dotter et al. 2008), BHAC-15 (Baraffe et al. 2015), and SPOTS models (Somers et al. 2020, for different spot coverage fractions) that are consistent within a 95 per cent confidence level ( $\chi^{2}<6$ for 2 degrees of freedom) with the luminosity and $T_{\text {eff }}$ of V1298 Tau (upper loci) and its candidate co-moving companion 2M0405 (lower loci). Each colour represents those interpolated models with $T_{\text {eff }}$ and $L_{\text {bol }}$ consistent with observations. The coloured circles are the best-fitting (minimum $\chi^{2}$ ) values labelled with the corresponding age. The colour-coded horizontal bars represent and are labelled by the overlap in $2 \sigma$ model ages, i.e. the allowed age range if the stars are co-eval. The dotted line is the minimum age of the co-moving Group 29 described by Luhman (2018), while the vertical dashed magenta lines are the estimated age range of the co-moving Taurus D3-South subgroup describe Krolikowski et al. (2021). The discontinuity in the Dartmouth Magnetic fits for 2M0405 is a result of a gap in model parameter coverage.

coeval since the collapse time of dense molecular cloud cores is $\sim 10^{5}$ yr (Chevance et al. 2020). We therefore searched for possible companions to V1298 Tau by identifying stars with similar parallaxes and proper motions in the Gaia EDR3 data base out to a radius of $1 \mathrm{deg}$. Only two other stars have parallaxes within $5 \sigma$ of that of V1298 Tau and similar proper motions: HD 284154, 98 arcmin away, and 2MASS J04051277+2007489, hereafter 2M0405, with a separation of 137 arcmin. Both stars were identified as significant X-ray emitters and possibly young and related to V1298 Tau by Poppenhaeger, Ketzer \& Mallonn (2021). HD 284154, was identified as a potential companion to V1298 Tau by Oh et al. (2016), but (ironically) ruled out as a Taurus member by Gagné et al. (2018). It does not have a published RV, but if this were to prove to be the same as V1298 Tau then the space velocity difference could be as small as $0.3 \mathrm{~km} \mathrm{~s}^{-1}$. 2M0405, which is 40 arcsec from HD 284154, does have a Gaia spectrometer measurement of RV $\left(12.64 \pm 0.93 \mathrm{~km} \mathrm{~s}^{-1}\right)$ which is within error of that of V1298 Tau. Under the assumption that the two stars are at the same distance the difference in velocity is $2.0 \pm 0.89 \mathrm{~km} \mathrm{~s}^{-1}$, a $2.2 \sigma$ level of significance. The formal probability that the velocity is less than the escape speed at the projected separation $\left(0.38 \mathrm{~km} \mathrm{~s}^{-1}\right)$ is $0.03 .^{2}$ Moreover, the Gaia Reduced Unit Weighted Error (RUWE), a measure of the astrometric error, is 1.66 which means that the star itself is likely to be a binary (Belokurov et al. 2020) and its proper motion and hence velocity errors are likely to be larger than reported in EDR3. Obtaining echelle spectra and precision RVs and AO imaging is clearly a high priority

\footnotetext{
${ }^{2}$ These calculations assume Gaussian, independent errors which is not the
} case for Gaia astrometry. 
to establish whether the system is gravitationally bound and whether 2M0405 is indeed a binary is clearly a high priority.

We first analysed observations of this star assuming that it is a highcontrast binary, i.e. that the companion contributes negligibly to the total source flux. We fit stellar atmosphere models to the photometry of 2M0405 (APASS DR10, 2MASS Point Source Catalog, the ALLWISE catalog, and Gaia GBpRp), adopting a reddening of $E(B$ $-V)=0.024$ (David et al. 2019a). The best-fitting solar-metallicity BT-SETTL model with CIFIST solar abundances yielded $\chi^{2}=$ 2.44 for 11 degrees of freedom and $T_{\text {eff }}=3900 \mathrm{~K}$ and $\log g=$ 4.5. We compared stellar evolution models to the inferred $T_{\text {eff }}$ and luminosity of 2M040, yielding an age of 5-22.5 Myr. Next, we computed concordant ages as the overlap between the individual 95 per cent confidence intervals. These span much narrower ranges, with spot-free model ages spanning $8-10 \mathrm{Myr}$ and progressively more magnetic models ranging up to 22-27 Myr (horizontal bars in Fig. 2). The spot fraction $f_{c}$ and magnetic field of V1298 Tau are not known and could vary with stellar cycles. Morris (2020) model the $K 2$ Campaign 4 light curve of V1298 Tau to estimate $f_{S} \approx 9_{-2}^{+1}$ per cent, which would suggest a concordance age of $\approx 10$ $12 \mathrm{Myr}$ (assuming a similar $f_{S}$ for 2M0405), significantly younger than the $23 \pm 4$ Myr estimated by David et al. (2019a). But it has long been known that such estimates suffer from model degeneracies (e.g. Luger et al. 2021) and higher spot fractions and thus older ages are possible. Furthermore, since 2M0405 is a binary in which each component is less luminous than the sum, its model ages could be older. Concordance between the age estimates for the two stars is maximized by artificially reducing the luminosity of $2 \mathrm{M} 0405$ by 20 per cent, and becomes 10-15 Myr. Better constrains on the age of V1298 Tau will require precise characterization, binary identification, and isochrone fitting of 2M0405 and HD 284154, any other co-moving neighbours, and members of the D3-South sub-group and Group 29.

\subsection{Rossiter-McLaughlin effect and Doppler-shadow analysis}

In order to constrain the stellar obliquity of V1298 Tau relative to the normal of planet b's orbit plane, we analysed its RV sequence during the transit observed by IRD. The relative RVs during the transit are plotted in Fig. 3 and suggests an anomaly typical of the RossiterMcLaughlin (RM) effect for transiting planets (e.g. Winn et al. 2005). However, a fit to the observed RVs, in which all the relevant parameters were allowed to vary freely, resulted in poor constraints on the fitting parameters. Due to the lack of out-of-transit baseline data on the transit night, the MCMC analysis performed poorly, leading to degeneracy among the system parameters including the RV semi-amplitude $K$, which accounts for any change in RV during the transit but unrelated to the transit itself, $v \sin i$, and the projected stellar obliquity $\lambda$ (see the green dotted line in Fig. 3 , representing the best-fitting RV model without any priors on the fitting parameters). Moreover, V1298 Tau is known to exhibit large activity-induced RV noise ('jitter') on the time-scale of stellar rotation (David et al. $2019 b$ ), and therefore RV data taken on other nights were not helpful enough to determine the RV baseline during the transit. We thus opted to impose Gaussian priors on $v \sin i$, the scaled semimajor axis $a / R_{s}$, full transit duration $T_{14}$, and $K$ as $v \sin i=23 \pm 2 \mathrm{~km} \mathrm{~s}^{-1}, a / R_{s}=$ $27.0 \pm 1.1, T_{14}=6.42 \pm 0.13 \mathrm{~h}$ (David et al. 2019b, a), and $K=$ $0 \pm 400 \mathrm{~m} \mathrm{~s}^{-1}$, respectively. Beichman et al. (2019) obtained $K=$ $10 \pm 48 \mathrm{~m} \mathrm{~s}^{-1}$ for V1298 Tau b, but since it was based on a relatively short-term RV monitoring, we conservatively imposed a weak prior whose width is twice as large as the size of the reported RV jitter (200 $\mathrm{m} \mathrm{s}^{-1}$; David et al. 2019b).

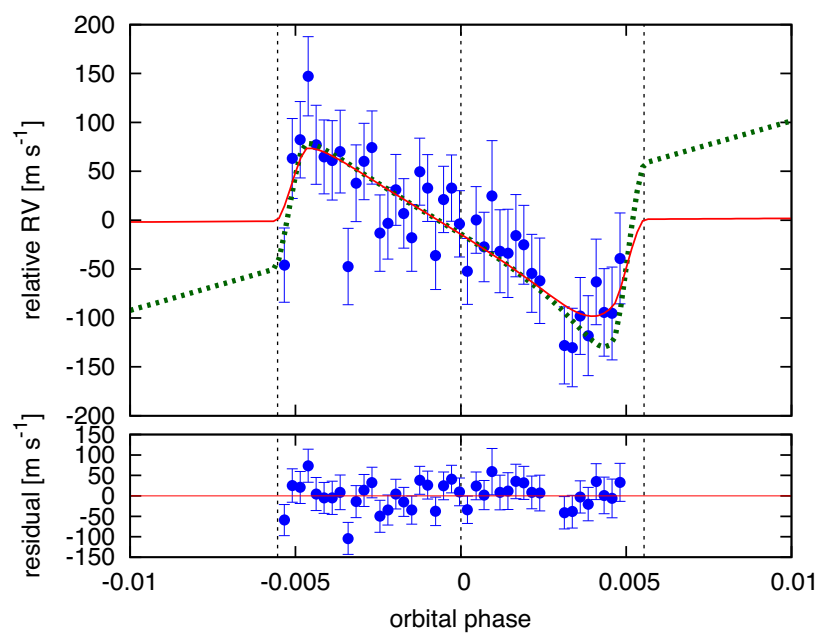

Figure 3. Relative RV of V1298 Tau on UT 26 September 2020. The bestfitting RM plus RV-baseline model, in which we imposed priors on $K$ and $v \sin i$, is drawn by the red solid line. The green dotted line indicates the bestfit model, in which no priors are assumed for $K$ and $v \sin i$ (those parameters show very broad posterior distributions; see the text). The lower panel plots the RV residual from the best-fitting model. The three vertical dashed lines in each panel represent the transit ingress $\left(T_{1}\right)$, centre $\left(T_{c}\right)$, and egress $\left(T_{4}\right)$ times of V1298 Tau b.

We modelled the RV anomaly during the transit using the analytic RM formula in Hirano et al. (2011), and implemented the MCMC analysis to estimate the obliquity $\lambda$. In addition to the above fitting parameters, we allowed the RV zero-point $\gamma$ for our RV data set and the mid-transit time $T_{c}$ to float, for the latter of which we imposed Gaussian prior based on the predicted ephemeris (i.e. $T_{c}=$ $2459119.0231 \pm 0.0012$ day in BJD). Implementing the MCMC simulations, we obtained $K=(-0.3 \pm 3.2) \times 10^{2} \mathrm{~m} \mathrm{~s}^{-1}, v \sin i=$ $23.4 \pm 1.9 \mathrm{~km} \mathrm{~s}^{-1}$, and $\lambda=15_{-16}^{+15}$ degrees, implying a low projected obliquity for V1298 Tau. The best-fitting RV curve with the analytic RM formula is drawn by the red solid line in Fig. 3 .

To further investigate the alignment between the rotation of V1298 Tau with the orbit of 'b', we investigated the variation in the line profile during the transit, performing the 'Doppler-shadow' analysis as in Hirano et al. (2020b) and Gaidos et al. (2020b). This technique directly detects and models the profile-variation of the cross-correlation function (CCF) of the observed spectra, caused by the partial occultation of the rotationally broadened stellar line profile. Following Hirano et al. (2020b), we cross-correlated each observed IRD spectrum of V1298 Tau against a theoretical template of V1298 Tau (Coelho et al. 2005), after correcting for telluric features. The resulting $\mathrm{CCF}$ s were aligned to the same reference RV taking into account the barycentric corrections of the Earth's motion. Usually, the mean 'out-of-transit' CCF is subtracted from the individual CCFs to highlight the residual $\mathrm{CCF}$ variation, but since we lack such out-of-transit frames taken on the same night, we median-combined all the CCFs that were taken during the transit. We then subtracted this combined CCF from each CCF finally to obtain the residual CCF map as shown in the left panel of Fig. 4. While the residual CCF map includes a correlated-noise pattern, likely caused by temporally evolving stellar surface inhomogeneity, the residual CCF map indicates a relatively clear shadow of the transiting planet that is traversing the stellar disc from the red-shifted edge to the blue-shifted edge. The symmetric pattern of the shadow is indicative of the prograde, aligned orbit of V1298 Tau b. 

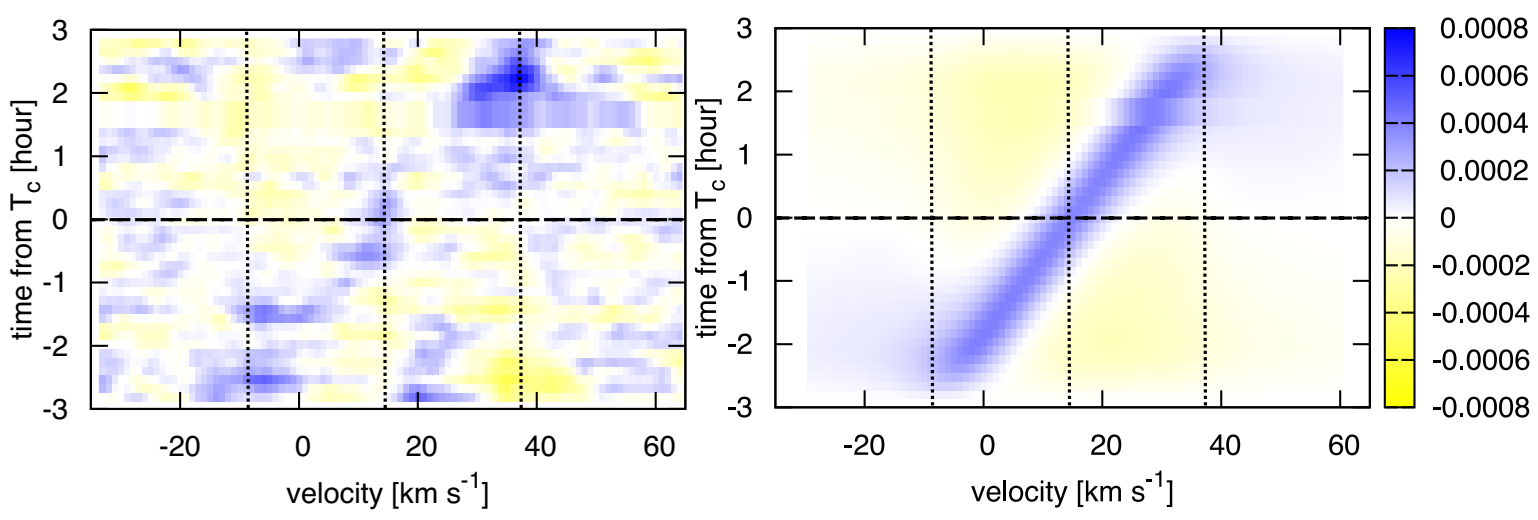

Figure 4. Residual CCF maps for the observed IRD spectra (left) and best-fitting theoretical model (right). The three vertical lines for each panel indicate the approximate $\mathrm{CCF}$ centre and its $\pm 23 \mathrm{~km} \mathrm{~s}^{-1}(\approx v \sin i)$, corresponding to the line edges. The horizontal dashed line represents the mid-transit time $T_{c}$.

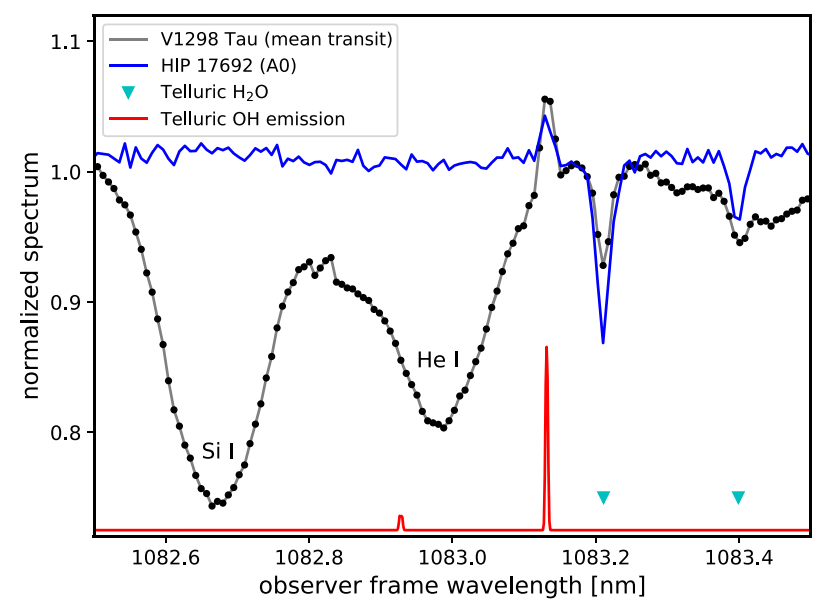

Figure 5. Average of spectra in the vicinity of the He I $1083 \mathrm{~nm}$ triplet of V1298 Tau obtained during the transit of 'b' with wavelengths in the frame of the observer. The relatively featureless spectrum of an A0-type star HIP 17692 is also shown. Blue triangles are telluric $\mathrm{H}_{2} \mathrm{O}$ lines cataloged by Breckinridge \& Hall (1973) and the red curve is a model of sky emission (i.e. OH) based on Noll et al. (2012) and Jones et al. (2013).

We estimated the projected obliquity $\lambda$ by fitting this residual CCF map. The detail of the analysis is given in Hirano et al. (2020b). In brief, we generated a number of theoretical CCFs by making 'mock' IRD spectra during V1298 Tau b's transit, and the observed residual CCF was fitted using those theoretical models with $\lambda$ and other relevant parameters being optimized. Following the exact procedure as for the observed data, we combined the theoretical CCF models during the transit and subtracted the combined model from individual CCF models when comparing the observed residual CCF map with the theoretical one. This analysis yielded $\lambda=2_{-4}^{+12}$ degrees, which is fully consistent with and more constraining than the RV analysis. The best-fitting theoretical residual CCF model is shown in the right-hand panel of Fig. 4.

\section{$3.51083 \mathrm{~nm}$ Orthohelium triplet}

The He I triplet at $\approx 1083 \mathrm{~nm}$ appears in a single order (1075.67$1089.84 \mathrm{~nm}$ in air) of IRD spectra. Spectra obtained during the transit of ' $b$ ' and those obtained for an independent RV monitoring programme (C. Beichman personal communication) were evaluated based on overall signal and interference of telluric emission $(\mathrm{OH})$ and absorption lines $\left(\mathrm{H}_{2} \mathrm{O}\right)$ with the He I triplet. (IRD spectra are obtained through a single fibre on the target and this precludes simultaneously measurement of the sky.) We retained 53 spectra spanning $10.2 \mathrm{~d}$, including all spectra obtained during the transit, from $1.1 \mathrm{~d}$ pre- $T_{c}$ to 9.1 days post- $T_{c}$, with adequate signal and with an observer Doppler shift $>0 \mathrm{~km} \mathrm{~s}^{-1}$ such that telluric interference was not a concern. Spectra were shifted to the rest frame of V1298 Tau. Potential signals from the planet in spectra obtained during transit were corrected for the planet's orbital motion (assumed circular) by dividing these spectra by a mean out-of-transit spectrum, shifting the residual spectra by $2 \pi R_{*} t /(P \tau)=2.74(t / \tau) \mathrm{km} \mathrm{s}^{-1}$, where $t$ is the time relative to $T_{c}$ and $\tau$ is the transit duration, and adding the Doppler-corrected residuals back to the out-of-transit reference.

Fig. 5 plots the mean observed spectrum of V1298 Tau obtained during the transit, as well as the spectrum of the A0 star HIP 17692, along with the positions of known telluric lines due to $\mathrm{H}_{2} \mathrm{O}$ (in absorption) and $\mathrm{OH}$ (in emission). The rapid rotation of V1298 Tau partially blends the $\mathrm{He}$ I triplet with a nearby $\mathrm{Si}$ I line. The two $\mathrm{H}_{2} \mathrm{O}$ lines as well as the stronger, redder $\mathrm{OH}$ line are readily apparent in the spectra while the weaker $\mathrm{OH}$ line is not. Importantly, there is no evidence for additional telluric lines.

Fig. 6 shows He I spectra in the rest frame of the star, and compares the average of spectra obtained during the transit night, and the preceding. The spectra during transit are also binned according to whether they were obtained during the first or second half. There is (i) elevated absorption during the transit relative to one day before the transit; (ii) a trend of decreasing absorption and return to pre-transit absorption during the transit; and (iii) anomalously high absorption in the $N=2$ spectra obtained on the night following the transit. In contrast, the neighbouring Si I line is unchanged throughout this interval. All the spectra obtained outside of transit are plotted individually in Fig. 7. All are consistent except for the two spectra obtained on the following night.

Fig. 8 is a 'tomographic' plot of the deviations of the He I line during the transit of ' $b$ ' relative to the pre-transit profile (mean of observations on the previous night). Red areas are ones of decreased signal (increased absorption) and blue areas represent increased signal (decreased absorption). This plot clearly shows the increase in the strength of both the singlet and doublet which make up the He I triplet which begins sometime between observations of the previous night and the start of observations around transit first contact (T1), the decline in the absorption over the night, the anomalous absorption 


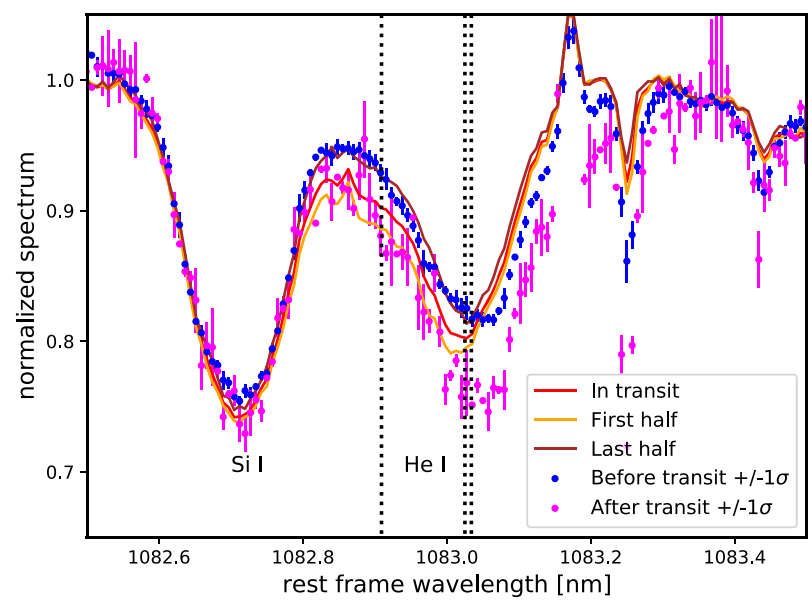

Figure 6. Spectra of V1298 Tau in the frame of the star, showing variation in the He I line before, during, and after the transit of ' $b$ ' (but no significant variation in the neighboring Si I line. Error-bars show the standard deviation among the multiple spectra obtained on nights previous to and following the transit night. Also plotted are the means of spectra obtained during the first half and last half of the transit.

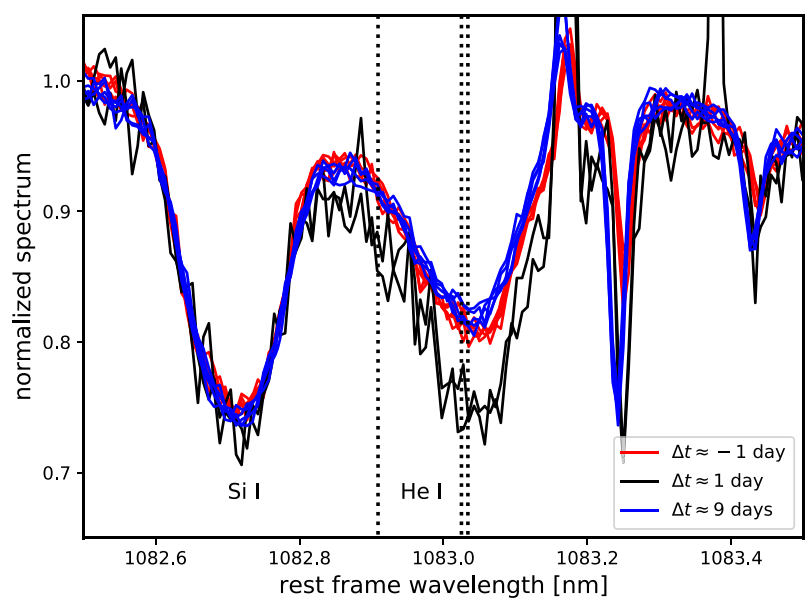

Figure 7. All spectra with acceptable signal level obtained outside of the transit of ' $b$ ' where the observed $\mathrm{RV}$ is $>0$ and the telluric $\mathrm{OH}$ line does not interfere with the He I line. The spectra are colour coded according to the approximate time relative to $T_{c}$.

on the following night, and the return to pre-transit level eight nights later. There also appears to be a slight increase in emission at the longer wavelength side of the triplet. Variability in the telluric lines of $\mathrm{OH}$ and $\mathrm{H}_{2} \mathrm{O}$, well separated from the $\mathrm{He} \mathrm{I}$ are evident, as well as the lack of variability in the $\mathrm{Si}$ I line.

We measured the total equivalent width (EW) of the He I triplet, the neighbouring Si I and Mg I lines (1082.7089 and $1081.1076 \mathrm{~nm}$ ), and the Paschen $\beta$ line of $\mathrm{H}$ I at $1281.8072 \mathrm{~nm}$. Lines were modeled with Voigt profiles, line constants were obtained from the NIST Atomic Line Database, and the FWHM of the Gaussian component of the Voigt profile was fit as a free parameter. Two additional free parameters in the He I fit were the ratio of the singlet to the doublet line strength (to allow for variation due to regions on the star's surface where the lines are optically thick and thus the ratio can change) and an overall Doppler shift relative to the NIST line centre (to account
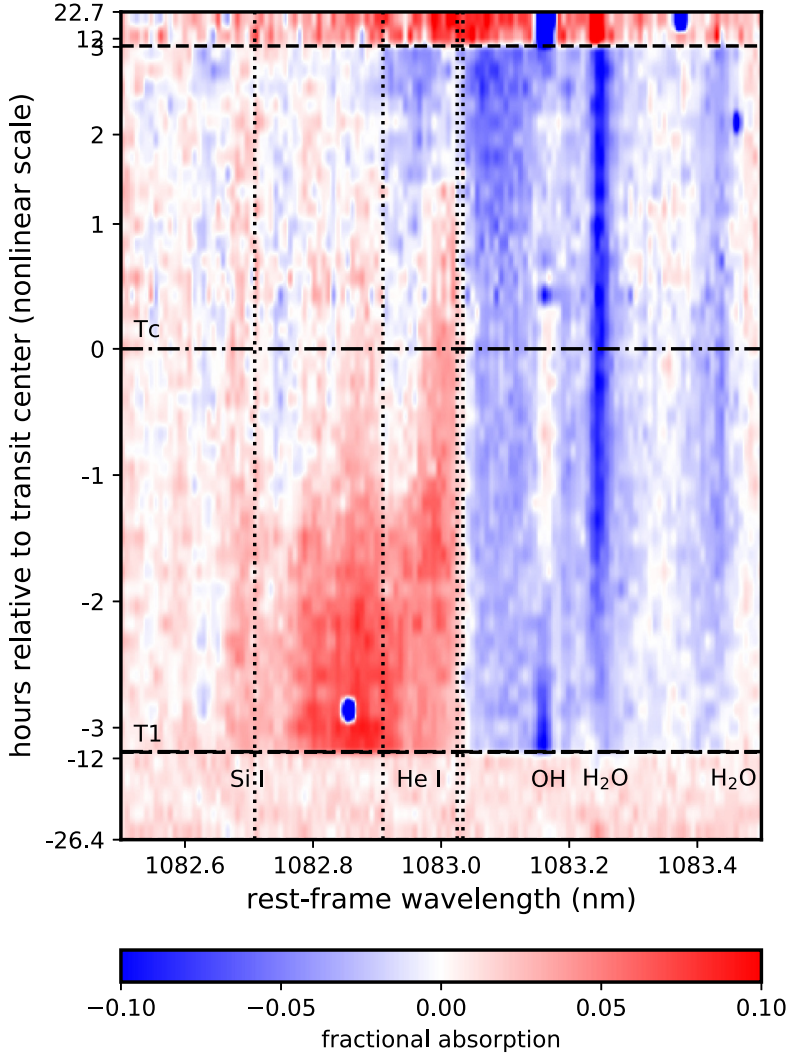

Figure 8. 'Tomography' of the differential normalized spectrum of V1298 Tau in the vicinity of the He I triplet relative to the before-transit mean. Red indicates less signal (greater absorption) and blue indicates more signal (less absorption or greater emission). Each observation is represented by a separate row of pixels and since monitoring was not continuous during this period the vertical time-axis is also not uniform or continuous. Horizontal dashed lines separate observations on different nights, while the dot-dashed line marks the beginning of the transit $\mathrm{T} 1$ and transit centre $T_{c}$ (observations ceased prior to the end of transit). Vertical dotted lines mark the individual $\mathrm{He}$ I line centres and a neighbouring $\mathrm{Si}$ I line. Telluric lines of $\mathrm{OH}$ and $\mathrm{H}_{2} \mathrm{O}$ are also marked. The Doppler shift of the planet's rest frame is negligible on this scale.

for an uneven distribution of active regions) - a significant source of He I absorption - on the rotating surface of the star.

The top panel of Fig. 9 shows the EW versus time obtained during the night of the transit, the preceding and following nights, and nine nights after the transit. While the He I EW measured $1 \mathrm{~d}$ before and $9 \mathrm{~d}$ after the transit are consistent, the EW varies by $\sim 50$ per cent during the transit, decreasing from 0.047 to $0.028 \mathrm{~nm}$. The EW of other lines in the neighbourhood of $1083.2 \mathrm{~nm}$ vary by much less. This variation is not correlated with the overall signal in the spectral order (second panel of Fig. 9), although the high He I EW values on the night after the transit were obtained with lower signal during cloudy conditions. The FWHM of the He I line (third panel of Fig. 9) exhibits a slight decrease during the transit and returns to the pre-transit value, indicating that the transit-associated absorption is broader than the out-of-transit line. During the transit, the best-fitting line centre (bottom panel of Fig. 9) is blue-shifted by $\sim 4 \mathrm{~km} \mathrm{~s}^{-1}$ during the transit relative to outside the transit; this is clearly seen in the blue side of the He I line profile in Fig. 5.

Possibly, the observed changes in He I EW could have been due to variation in the intensity of the known, weaker $\mathrm{OH}$ line 


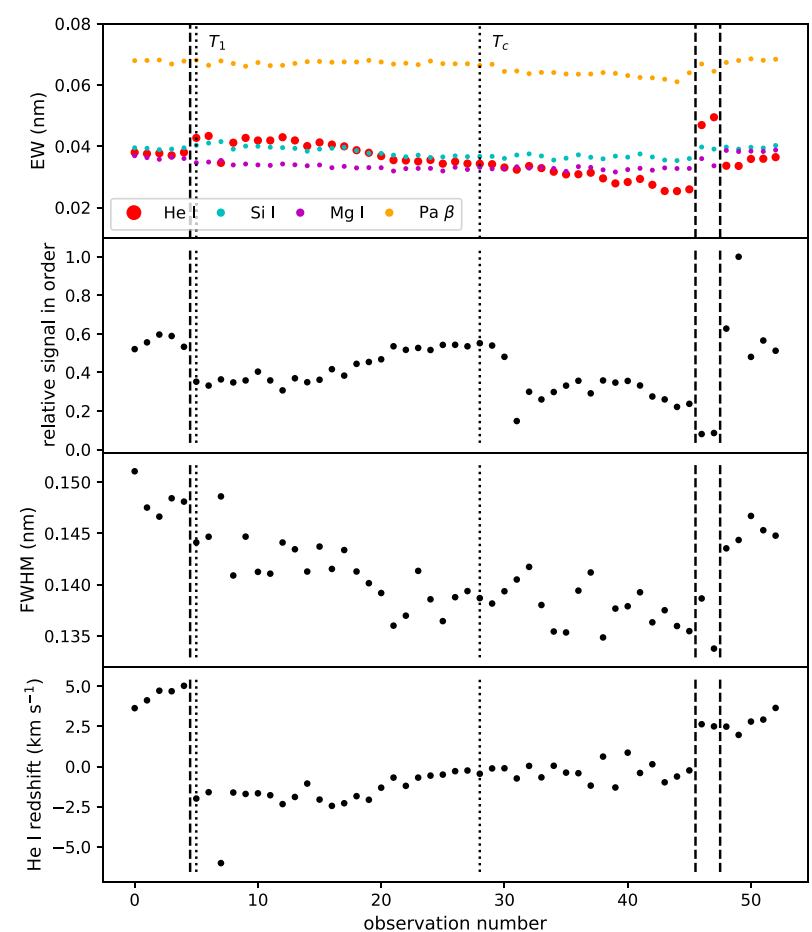

Figure 9. Top row: EW of the He I triplet, neighboring Si I and Mg I lines, and the Paschen $\beta$ line of $\mathrm{HI}$ (a potential indication in stellar activity). All data with sufficient signal and non-interference with tellurics are plotted. Dashed lines separate different nights and the beginning $\left(T_{1}\right)$ and mid-point $\left(T_{c}\right)$ of the transit of ' $b$ ' are marked by dotted lines. Second row: Relative signal in the spectral order containing the He I triplet. Third row: FWHM of the He I triple and $\mathrm{Mg}$ I and $\mathrm{Si}$ I lines as a fit parameter in the Gaussian component of a Voigt line profile model. Bottom row: Red-shift in the He I triplet relative to the NIST values as a fitting term in the line profile model.

(Fig. 5) or an unknown $\mathrm{H}_{2} \mathrm{O}$ line. However, the observed variation appears much broader than that observed for other $\mathrm{OH} / \mathrm{H}_{2} \mathrm{O}$ lines and nothing analogous appears in the relevant wavelength range of either V1298 Tau or the A0-type telluric reference star HIP 17692. Error in determination of the continuum level would manifest itself in the inferred EW, and is a leading contender to explain the drift in the $\mathrm{Mg}$ I and $\mathrm{Si}$ i lines, but this would affect all neighbouring lines equally and thus cannot explain the behaviour of the $\mathrm{He}$ I line. Variation in the instrument profile is minimal based on the FWHM behaviour as well as the small $\left(10-12 \mathrm{~m} \mathrm{sec}^{-1}\right)$ instrumental drift (highly correlated with FWHM) during the transit and to first order is unlikely to explain this variation.

Astrophysical explanations for the variation during the transit include changes in the line as a result of stellar activity, i.e. a flare and rotation of active regions into and out of the visible disc of the star. V1298 Tau is a rapidly rotating (2.87-day), active star and these effects are expected to be enhanced relative to middle-aged stars. Strong He I line emission and/absorption can also arise from inner disc winds or accreting disc gas (Edwards et al. 2003; Fischer et al. 2008; Kwan \& Fischer 2011), but V1298 Tau lacks the infrared excess of a T Tauri-like disc or any sign of accretion (David et al. 2019a).

He I emission from a flaring region fills in the line core and produces a weakening both of He I at $1083 \mathrm{~nm}$ and the D3 line at $587.6 \mathrm{~nm}$ that tracks other chromospheric emission indicators such as $\mathrm{H} \alpha$ (Johnson et al. 2021; Schmidt et al. 2012). However, the change in the steady decline in EW over the $6 \mathrm{~h}$ observation interval is inconsistent with the increase expected in the declining phase of a flare, and the time-scale is inconsistent with the more rapid ( $\lesssim 1 \mathrm{~h})$ rise time of flares. ${ }^{3}$ Emission is also associated with Doppler shifts of $10 \mathrm{~s}_{\text {of } \mathrm{km} \mathrm{s}^{-1}}$ (Johnson et al. 2021) which we do not observe.

The rotation period of V1298 Tau has been determined to be $2.87 \mathrm{~d}$ and thus over the $6 \mathrm{~h}$ observing window approximately $30^{\circ}$ of longitude appears and disappears, i.e. 1/6th of the visible disc. A 50 per cent variation would require a fortuitous arrangement of active regions on the stellar surface. Notably, values well before and after transit agree within a few per cent (upper left panel of Fig. 9). Feinstein et al. (2021) found no variation in the He I D3 line during a 6-h observation of a transit of the 'c' planet, but did detect a steady 6 per cent decrease in $\mathrm{H} \alpha$ absorption which they attributed to rotation or variation of active regions on the star, and well within the range observed among other young stars (Fig. 7). Fuhrmeister et al. (2020) found no evidence for rotational variability of the $1083 \mathrm{~nm}$ He I line among M dwarfs (including a few rapid rotators), although this might be due in part to limitations of their sample, and of course V1298 Tau is young and much more active than the typical middle-aged $\mathrm{M}$ dwarf. While we cannot rule out a stellar cause, other observations cast doubt on this as the explanation for a 50 per cent variation in He I.

We further investigated the intrinsic variability of the stellar He I line by analysing all spectra with sufficient signal, regardless of telluric contamination, by simultaneously fitting the $\mathrm{OH}$ and $\mathrm{H}_{2} \mathrm{O}$ lines with Gaussian profiles with a fixed FWHM of $0.0155 \mathrm{~nm}$ (the instrument resolution) but variable equivalent width and line center, along with the He I triplet and blended Si I line. While these fits capture the overall strength of the lines, the limited spectral sampling and departures of the line profiles from idealized models degrade the fidelity of the fits. The top panel of Fig. 10 shows that there is apparent variability of a magnitude similar to that observed during the transit, but this occurs when the observer-frame RV of the star (third panel of Fig. 10) means that the He I line is contaminated by telluric $\mathrm{OH}$ and $\mathrm{H}_{2} \mathrm{O}$ lines, and the $\mathrm{He} \mathrm{I}$ EW variation is largely correlated with the strength of those lines (bottom row of Fig. 10). In particular, the apparent drop in He I EW on nights 3-5 and 10-15 relative to nights 1-2 occurs when the interfering telluric lines are significantly stronger. The increasing trend over $3 \mathrm{~h}$ on night 1 suggests that the star could be responsible for the variation we seen during the transit on night $7 .{ }^{4}$

The alternative astrophysical explanation is an affect associated with the 'b' planet, but this is difficult to conclusively demonstrate with these data. The occultation of individual active regions by the transiting planet will produce variation, but since the transit depth is 0.6 per cent (David et al. 2019a), these regions would have to occupy only $\sim 0.3$ per cent of the stellar disc, which is completely inconsistent with the observed strength of the He I line. Occultation by an extended He-containing cloud would seem to be required, but the cloud would have to precede the planet in its orbit. Moreover, the anomalous absorption on the night following the transit would remain to be explained. The formal uncertainty in $T_{c}$ is only $\pm 2 \mathrm{~min}$.

V1298 Tau hosts multiple planets: could another of these be responsible? Using the linear ephemerides for the V1298 Tau system

${ }^{3}$ Flare-induced dimming, perhaps a result of electron-collisional ionization of triplet He I has been observed on the Sun (Kerr et al. 2021) but is low contrast and localized and unlikely to be responsible.

${ }^{4}$ No known planets transited during night 1 and the next transit (of ' $c$ ') started about $16 \mathrm{~h}$ after these observations ended. 


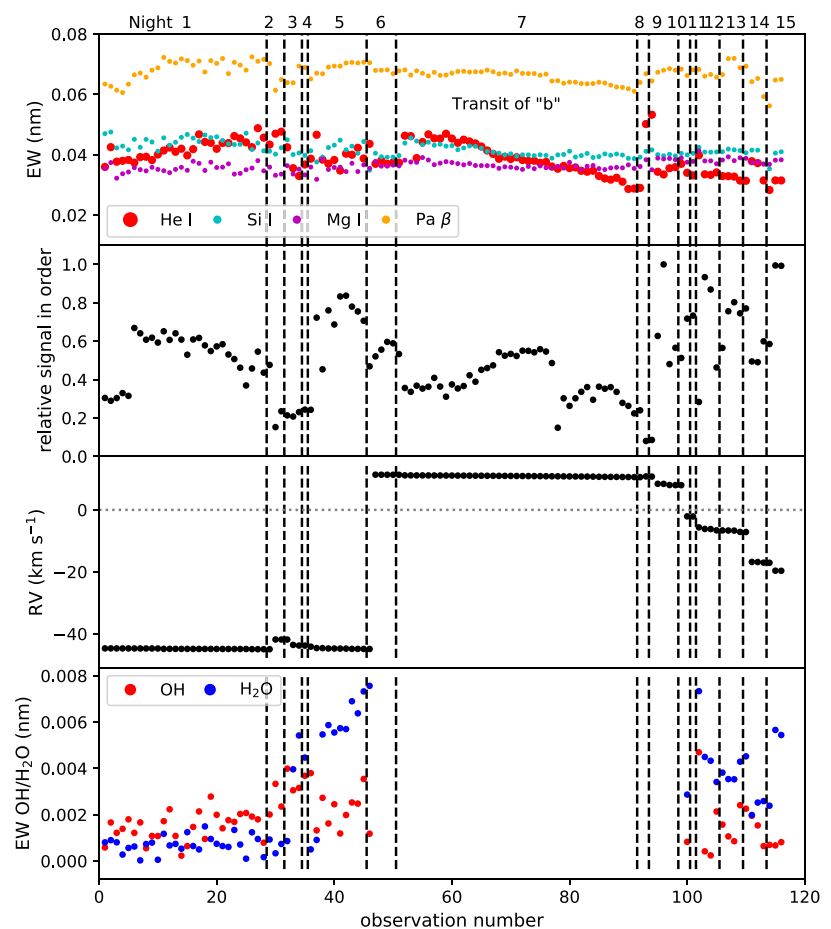

Figure 10. Similar to Fig. 9 but for all spectra with acceptable signal, along with diagnostics of spectrum quality. Data are plotted in simple temporal order, with vertical dashed lines separating different nights, which are numbered at the top. Top row: EW of the He I triplet, neighbouring Si I and $\mathrm{Mg}$ I lines, and the Paschen $\beta$ line of $\mathrm{HI}$ (an indicator of stellar activity) determined by fitting Voigt line profiles. Second row: Relative level of signal in the order containing the He I triplet. Third row: Observer-frame RV of the star, which determines interference by tellurics. In spectra obtained with RV $<0$ (below the dotted grey line) the $\mathrm{He}$ I triplet is contaminated by telluric $\mathrm{OH}$ and/or $\mathrm{H}_{2} \mathrm{O}$ lines. Bottom row: EW of the $\mathrm{OH}$ (red, emission) and $\mathrm{H}_{2} \mathrm{O}$ (blue, absorption) lines estimated by fitting Gaussian line profiles simultaneously with stellar lines. In spectra obtained with $\mathrm{RV}>0$, the $\mathrm{OH}$ and $\mathrm{H}_{2} \mathrm{O}$ are outside the fitting region and EWs are not plotted.

(Livingston et al. in preparation), a transit of 'c' occurs with a $T_{c}$ at $\mathrm{BJD}=2459118.3044$ (UT 19:18 on 25 September), $17.25 \mathrm{~h}$ before that of 'b', and a transit of ' $d$ ' has a $T_{c}$ at BJD $=2459118.6915$ (UT 4:36 on 26 September), only $7.96 \mathrm{~h}$ before $( \pm 8 \mathrm{~min})$. The transit of ' $d$ ' ends less than two hours before the beginning of our observations for the transit of ' $b$ '. This raises the possibility that the declining He I absorption observed during the transit of ' $b$ ' is actually associated with the prior transit of ' $d$ '. The radius of ' $d$ ', $6.4 \pm 0.4 R_{\oplus}$, is only marginally larger than that of ' $c$ ' $\left(5.6 \pm 0.3 R_{\oplus}\right)$, and both almost certainly have low-molecular weight (H/He-rich) envelopes. Why the more irradiated ' $c$ ' has little or no mass-loss (Feinstein et al. 2021), but 'd' might is unclear, but one possibility is simply that the yet-to-measured mass and thus gravity of ' $\mathrm{d}$ ' is much lower than that of 'c'.

\section{SUMMARY AND DISCUSSION}

The study of very young planetary systems such as that of V1298 Tau offer insight into the time-scales and mechanisms of planet formation and evolution that shape the exoplanet populations that large surveys such as Kepler, TESS, and RV surveys have revealed. One outstanding question is whether planets on close-in orbits formed close to their host star, migrated inward via torques from the natal disc, or were scattered inwards by gravitational interaction with another planet or companion star. The last scenario predicts that the normal of the planetary orbit plane could be significantly inclined to the stellar rotation axis, particularly at early times before tidal torques from the star could re-align the orbits. The observations of Feinstein et al. (2021) and our work presented here that the projected obliquity of the 'c' and ' $b$ ' planets, respectively, is small (and possibly zero) point to an origin involving the protoplanetary disc.

The low stellar obliquity for the V1298 Tau system is in line with the earlier results of obliquity measurements for young systems (e.g. Dai et al. 2020; Zhou et al. 2020; Gaidos et al. 2020b; Hirano et al. 2020b). This is also consistent with the empirical finding that cool exoplanet hosts ( $T_{\text {eff }} \lesssim 6250 \mathrm{~K}$ ) generally have low stellar obliquities (Winn et al. 2010; Albrecht et al. 2012); this empirical law was found for giant planets $\left(\gtrsim 5 R_{\oplus}\right)$ like V1298 Tau b. The number of obliquity measurements for young exoplanet systems is still limited $(<10)$, and further measurements for young exoplanets covering a wide range of stellar types would enhance our understanding on the origin and dynamical history of close-in exoplanets.

Poppenhaeger et al. (2021) use models of XUV-driven H/He escape to predict that the innermost (' $c$ ' and ' $d$ ') could experience significant or near-complete loss of their $\mathrm{H} / \mathrm{He}$ envelopes in $5 \mathrm{Gyr}$. Current mass-loss rates depend sensitively on the heavy-element ('core') content, but Poppenhaeger et al. (2021) estimate it could be as high as $0.8 \mathrm{M}_{\oplus}$ in $10 \mathrm{Myr}$. An important caveat is that they adopt the energy-limited formulation with a constant efficiency factor of 10 per cent, which Krenn et al. (2021) use detailed models of hydrodynamic escape to show can lead to errors of orders of magnitude in escape rates.

The EW of the He I triplet is elevated during a transit of ' $b$ ' relative to nights before and after the transit, and shows a steady decreasing trend during the transit. This does not seem to be a product of the instrument, telluric effects, or our analysis. It is difficult to understand this in terms of a surrounding and/or trailing cloud of neutral gas around ' $b$ ', but our observations begin only $1 \mathrm{~h}$ after the transit of 'd', a smaller (and presumably less massive) planet on an interior orbit. Multiplying the $\approx 2 \mathrm{~h}$ duration of the absorption event plus the $1 \mathrm{~h}$ between the end of the transit of ' $\mathrm{d}$ ' and the beginning of our observations, with the orbital velocity of ' $d$ ' yields a transverse extent of $\approx 7 \times 10^{5} \mathrm{~km}$, which is about 26 planet radii or 1.1 stellar radii. Due to its size, such a cloud would have to be optical thin in the He I line.

Vissapragada et al. (2021), also report no detection of He I absorption in a narrow He I-band filter associated with transits of ' $b$ ' but do find tentative evidence for absorption associated with partials transits of 'd' on UT 2020 October 8 and November 27. The corresponding EW of their measurement is $0.027 \pm 0.014 \mathrm{~nm}$, which is consistent with our measurement of an $\approx 0.19 \mathrm{~nm}$ change during the September 26 transit. They also obtained spectra containing asymmetric variation of the $\mathrm{He}$ I line on day-time-scales that could be driven by stellar flaring.

We estimate the expected He I absorption produced by an escaping atmosphere of $\mathrm{H} / \mathrm{He}$ from ' $b$ ' or ' $d$ '. The model described in detail elsewhere (Hirano et al. 2020b; Gaidos et al. 2020a, b), calculates the escaping atmosphere as an isothermal (temperature $T_{\text {wind }}$ Parker wind with a solar-like composition $(\mathrm{H} / \mathrm{He}=10.5)$. The spectral intensity from the star in the extreme ultraviolet (EUV) is a required input, since photons with $\lambda<50.4 \mathrm{~nm}$ are responsible for ionizing $\mathrm{He} \mathrm{I}$ and, via recombination, producing the neutral triplet state. Also needed is the intensity in the near ultraviolet (NUV), since photons with $\lambda<258.3 \mathrm{~nm}$ ionize the triplet state. The UV spectra of distant stars in molecular clouds are not possible to measure directly 

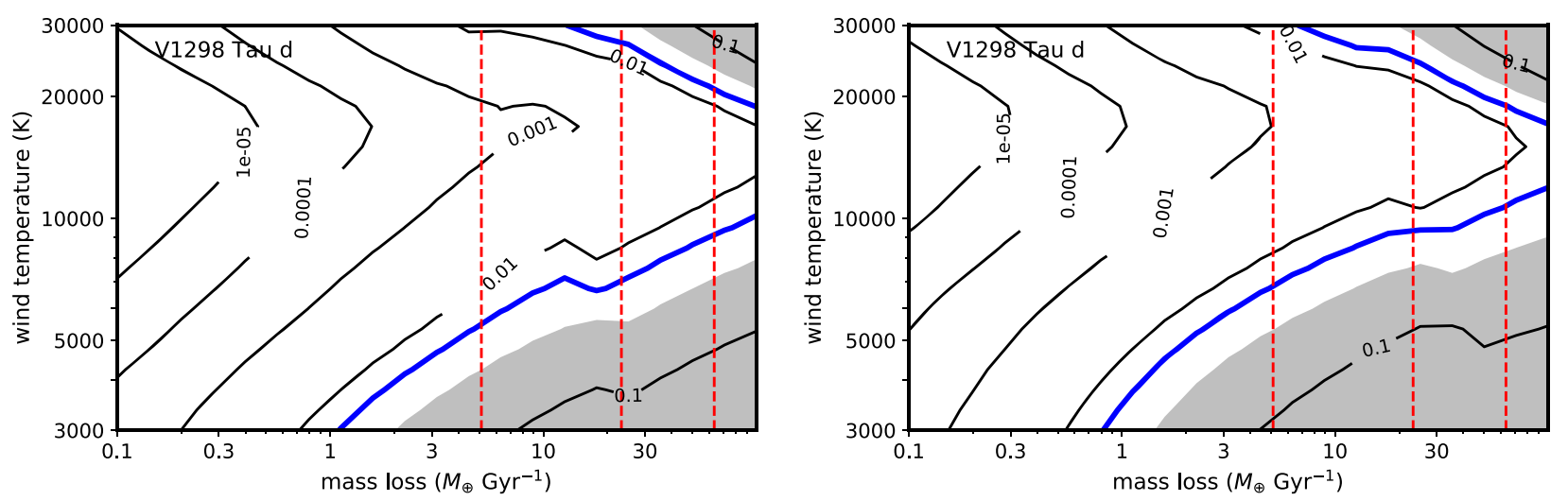

Figure 11. Contours of constant $\mathrm{He}$ ( (in nm) predicted by a model of atmospheric escape as an isothermal Parker wind from V1298 Tau b (left) and d (right). The thick blue contour represents the $0.019 \mathrm{~nm}$ change observed during the transit of ' $\mathrm{b}$ '. The grey region where EW $>0.04 \mathrm{~nm}$ can be excluded by our observations. The dashed red lines mark the low, medium, and high loss-rate estimates of Poppenhaeger et al. (2021).

due to absorption and scattering by intervening gas and dust and must be inferred from other measures such as X-rays. We adopted the spectrum of the young, active K2-type star Epsilon Eridani as reconstructed by the MUSCLES Treasury Survey (v. 2.2, France et al. 2016; Loyd et al. 2016; Youngblood et al. 2016). The spectrum was adjusted by the square of the radius ratio of V1298 Tau to Eps Eri. The EUV portion of the spectrum was also further adjusted so that the $0.01-1 \mathrm{kEV} \operatorname{EUV}(\lambda \lambda 12.39-123.9 \mathrm{~nm}$ ) luminosity is $5 \times 10^{30} \mathrm{ergs} \mathrm{s}^{-1}$, as estimated by Poppenhaeger et al. (2021). The latter was done by choosing a value for Ly $\alpha$ emission such that adjusting individual EUV wavelength ranges using the empirical relations between Ly $\alpha$ and EUV emission of Linsky, Fontenla \& France (2014) produces the Poppenhaeger et al. (2021) estimate for XUV luminosity. For a given planet radius, the predicted mass-loss rate depend sensitively on planet mass, but for a given escape rate the He I signal also depends only weakly on planet mass through the atmospheric scale height; for both ' $b$ ' and ' $d$ ' we assumed $10 M_{\oplus}$. Fig. 11 shows the predicted equivalent width in $\mathrm{nm}$ as a function of mass-loss rate and wind temperature, along with the observed change of $0.019 \mathrm{~nm}$ during the transit (thick blue line) and excluded levels $>0.04 \mathrm{~nm}$ (grey zone). Assuming that the observed change in He I EW is not associated with 'b', our observations rule out some or all of the range of mass loss estimated by Poppenhaeger et al. (2021), if $T_{\text {wind }}<7000 \mathrm{~K}$ (Fig. 11a). If instead the change is produced by an extended wind from 'd', the inferred escape rate (Fig. 11b) would support the predictions of Poppenhaeger et al. (2021) and result in the loss of an entire $\lesssim 1 \mathrm{M}_{\oplus} \mathrm{H} / \mathrm{He}$ envelope in $\sim 100$ Myr.

Our findings highlight the promise but also the challenges of observations of planets orbiting active young stars. In the case of V1298 Tau, our results and those of Feinstein et al. (2021) place limits on escape of the atmospheres of the b and c planets, respectively; extending this to the 'd' planet is a high-priority next step.

\section{ACKNOWLEDGEMENTS}

We thank Trevor David for providing an early version of the transit ephemerides, and Greg Feiden for Dartmouth magnetic stellar model tracks. EG was supported by NASA Grant 80NSSC20K0957 (Exoplanets Research Programme). This work is partly supported by JSPS KAKENHI Grant Numbers JP20K14518, JP19K14783, JP21H00035, JP18H05442, JP15H02063, and JP22000005. We used
NASA's Astrophysics Data System Bibliographic Services, the Centre de Données astronomiques de Strasbourg, NIST's atomic line data base, ASTROPY (Astropy Collaboration et al. 2013), and SCIPY (Virtanen et al. 2020).

\section{DATA AVAILABILITY}

All data used in this work are available from the authors or the Subaru SMOKA archive.

\section{REFERENCES}

Albrecht S. et al., 2012, ApJ, 757, 18

Astropy Collaboration et al., 2013, A\&A, 558, A33

Baraffe I., Homeier D., Allard F., Chabrier G., 2015, A\&A, 577, A42

Beichman C. et al., 2019, Res. Notes Am. Astron. Soc., 3, 89

Belokurov V. et al., 2020, MNRAS, 496, 1922

Breckinridge J. B., Hall D. N. B., 1973, Sol. Phys., 28, 15

Chevance M. et al., 2020, Space Sci. Rev., 216, 50

Coelho P., Barbuy B., Meléndez J., Schiavon R. P., Castilho B. V., 2005, A\&A, 443, 735

Currie T. et al., 2019, ApJ, 877, L3

Dai F. et al., 2020, AJ, 160, 193

Damasso M. et al., 2020, A\&A, 642, A133

David T. J. et al., 2019a, AJ, 158, 79

David T. J., Petigura E. A., Luger R., Foreman-Mackey D., Livingston J. H., Mamajek E. E., Hillenbrand L. A., 2019b, ApJ, 885, L12

Donati J. F. et al., 2016, Nature, 534, 662

Donati J. F. et al., 2020, MNRAS, 491, 5660

Dotter A., Chaboyer B., Jevremović D., Kostov V., Baron E., Ferguson J. W., 2008, ApJS, 178, 89

Edwards S., Fischer W., Kwan J., Hillenbrand L., Dupree A. K., 2003, ApJ, 599, L41

Feiden G. A., 2016, A\&A, 593, A99

Feinstein A. D., Montet B. T., Johnson M. C., Bean J. L., David T. J., GullySantiago M. A., Livingston J. H., Luger R., 2021, AJ, 162, 213

Fischer W., Kwan J., Edwards S., Hillenbrand L., 2008, ApJ, 687, 1117

France K. et al., 2016, ApJ, 820, 89

Froebrich D., 2005, ApJS, 156, 169

Fuhrmeister B. et al., 2020, A\&A, 640, A52

Gagné J. et al., 2018, ApJ, 856, 23

Gaia Collaboration et al., 2021, A\&A, 649, A1

Gaidos E. et al., 2020a, MNRAS, 495, 650

Gaidos E. et al., 2020b, MNRAS, 498, L119

Galli P. A. B. et al., 2019, A\&A, 630, A137 
Hirano T. et al., 2020a, PASJ, 72, 93

Hirano T. et al., 2020b, ApJ, 899, L13

Hirano T., Suto Y., Winn J. N., Taruya A., Narita N., Albrecht S., Sato B., 2011, ApJ, 742, 69

Itoh Y. et al., 2005, ApJ, 620, 984

Johansen A., Lambrechts M., 2017, Annual Review of Earth and Planetary Sciences, 45, 359

Johns-Krull C. M. et al., 2016, ApJ, 826, 206

Johnson E. N. et al., 2021, A\&A, 651, A105

Joncour I., Duchêne G., Moraux E., Motte F., 2018, A\&A, 620, A27

Jones A., Noll S., Kausch W., Szyszka C., Kimeswenger S., 2013, A\&A, 560, A91

Kerr G. S., Xu Y., Allred J. C., Polito V., Sadykov V. M., Huang N., Wang H., 2021, ApJ, 912, 153

Kotani T. et al., 2018, in Proc. SPIE. p. 1070211

Kraus A. L., Hillenbrand L. A., 2008, ApJ, 686, L111

Kraus A. L., Ireland M. J., Hillenbrand L. A., Martinache F., 2012, ApJ, 745, 19

Krenn A. F., Fossati L., Kubyshkina D., Lammer H., 2021, A\&A, 650, A94

Krolikowski D. M., Kraus A. L., Rizzuto A. C., 2021, AJ, 162, 110

Kruijer T. S., Burkhardt C., Budde G., Kleine T., 2017, Proceedings of the National Academy of Science, 114, 6712

Kwan J., Fischer W., 2011, MNRAS, 411, 2383

Lee D.-C., Halliday A. N., 1996, Science, 274, 1876

Linsky J. L., Fontenla J., France K., 2014, ApJ, 780, 61

Loyd R. O. P. et al., 2016, ApJ, 824, 102

Luger R., Foreman-Mackey D., Hedges C., Hogg D. W., 2021, AJ, 162, 123

Luhman K. L., 2018, AJ, 156, 271

Luhman K. L., Mamajek E. E., Allen P. R., Muench A. A., Finkbeiner D. P., 2009, ApJ, 691, 1265

Mendigutía I., Oudmaijer R. D., Schneider P. C., Huélamo N., Baines D., Brittain S. D., Aberasturi M., 2018, A\&A, 618, L9
Morris B. M., 2020, ApJ, 893, 67

Noll S., Kausch W., Barden M., Jones A. M., Szyszka C., Kimeswenger S., Vinther J., 2012, A\&A, 543, A92

Nortmann L. et al., 2018, Science, 362, 1388

Oh D. et al., 2016, ApJ, 831, L7

Ormel C. W., 2017, Formation, Evolution, and Dynamics of Young Solar Systems. Vol. 445, Astrophysics and Space Science Library. Springer, Cham, p. 197

Paunzen E., 2015, A\&A, 580, A23

Poppenhaeger K., Ketzer L., Mallonn M., 2021, MNRAS, 500, 4560

Schmidt S. J., Kowalski A. F., Hawley S. L., Hilton E. J., Wisniewski J. P., Tofflemire B. M., 2012, ApJ, 745, 14

Somers G., Cao L., Pinsonneault M. H., 2020, ApJ, 891, 29

Spake J. J. et al., 2018, Nature, 557, 68

Tamura M. et al., 2012, in Proc. SPIE. Vol. 8446, Ground-based and Airborne Instrumentation for Astronomy IV. p. $84461 \mathrm{~T}$

Thalmann C. et al., 2015, ApJ, 808, L41

Tody D., 1986, in Crawford D. L., ed., Proc. SPIE Vol. 627, Instrumentation in Astronomy VI. p. 733

Virtanen P. et al., 2020, Nature Methods, 17, 261

Vissapragada S. et al., 2021, AJ, 162, 222

Winn J. N. et al., 2005, ApJ, 631, 1215

Winn J. N., Fabrycky D., Albrecht S., Johnson J. A., 2010, ApJ, 718, L145

Youngblood A. et al., 2016, ApJ, 824, 101

Yu L. et al., 2017, MNRAS, 467, 1342

Zhou G. et al., 2020, ApJ, 892, L21

This paper has been typeset from a $\mathrm{T}_{\mathrm{E}} \mathrm{X} / \mathrm{LT} \mathrm{E} \mathrm{X}$ file prepared by the author. 